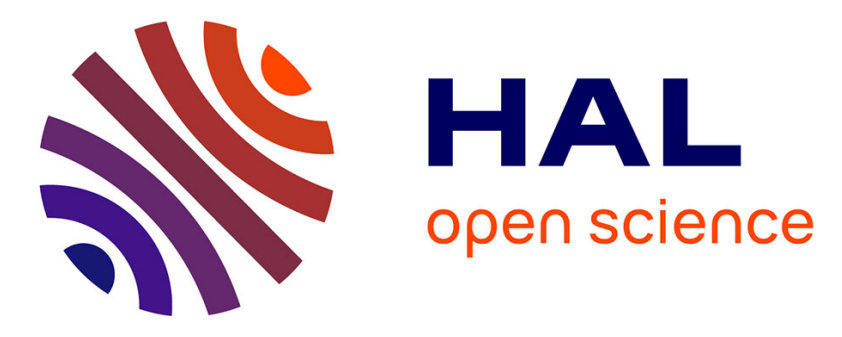

\title{
NMR and MRI observation of water absorption/uptake in hemp shives used for hemp concrete
}

Marine Fourmentin, Pamela Françoise Faure, Philippe Pelupessy, Vincent Sarou-Kanian, Ulrike Peter, Didier Lesueur, Stéphane Rodts, Daniel Daviller, Philippe Coussot

\section{To cite this version:}

Marine Fourmentin, Pamela Françoise Faure, Philippe Pelupessy, Vincent Sarou-Kanian, Ulrike Peter, et al.. NMR and MRI observation of water absorption/uptake in hemp shives used for hemp concrete. Construction and Building Materials, 2016, 124, pp.405-413. 10.1016/j.conbuildmat.2016.07.100 . hal-01784862

\section{HAL Id: hal-01784862 \\ https://hal-enpc.archives-ouvertes.fr/hal-01784862}

Submitted on 23 May 2018

HAL is a multi-disciplinary open access archive for the deposit and dissemination of scientific research documents, whether they are published or not. The documents may come from teaching and research institutions in France or abroad, or from public or private research centers.
L'archive ouverte pluridisciplinaire HAL, est destinée au dépôt et à la diffusion de documents scientifiques de niveau recherche, publiés ou non, émanant des établissements d'enseignement et de recherche français ou étrangers, des laboratoires publics ou privés. 


\title{
NMR and MRI observation of water absorption/uptake in hemp shives used for hemp concrete
}

\author{
M. Fourmentin ${ }^{1,5}$, P. Faure ${ }^{1}$, P. Pelupessy ${ }^{2}$, V. Sarou-Kanian ${ }^{3}$, U. Peter ${ }^{4}$, D. Lesueur ${ }^{5}$, S. \\ Rodts ${ }^{1}$, D. Daviller ${ }^{6}$, P. Coussot ${ }^{1}$ \\ 1. Université Paris-Est, Laboratoire Navier (ENPC-IFSTTAR-CNRS), Champs-sur-Marne, France \\ 2. Ecole Normale Supérieure, Département de Chimie, Paris, France \\ 3. Conditions Extrêmes : Matériaux à Haute Température et Irradiation, Orléans, France \\ 4. LHOIST Recherche et Développement, Nivelles, Belgium \\ 5. LHOIST France, Paris, France \\ 6. BCB Lhoist Sud Europe, Besançon, France
}

\begin{abstract}
Hemp concrete used as a construction material is known to provide excellent thermal insulation and hydric regulation, and prevents condensation. Initial water content in the hemp and water exchanges between hemp and binder play a major role in these processes. Here we study how hemp absorbs liquid water. In that aim we rely on ${ }^{1} \mathrm{H}$ NMR (Nuclear Magnetic Resonance) measurements which make it possible to distinguish water situated inside from water situated outside the hemp. Then, following the evolution of the distribution of NMR relaxation times we are able to quantify the effective amount of water entering the hemp as a function of time. We show that such a measure is better controlled than usual techniques such as weighing an immersed sample or the TC (Technical Committee) RILEM (Réunion Internationale des Laboratoires et Experts des Matériaux, systèmes de construction et ouvrages) method which cannot easily distinguish water inside hemp from water situated outside or wetting the external hemp surface. The water absorption in hemp occurs in two steps: about half the water enters the material in a time of the order of a minute, while the second half of water slowly penetrates over a time of the order of three days. Finally, from ${ }^{1} \mathrm{H}$ micro NMR imaging we show that the first step corresponds to water entering the pith while the second one corresponds to water diffusing in the wood part of the shiv.
\end{abstract}

\section{Introduction}

Current environmental problems lead us to develop more environmentally friendly materials. In particular new concretes are used that contain vegetal aggregate. Hemp shives are a byproduct resulting from the pulping of hemp stalks. They were originally used as animal bedding, and since the early 1990, they are also used as aggregates in hemp concrete in which the binder is often a mix of cement and hydrated lime. From a 'life-cycle assessment' perspective hemp construction materials are far more interesting: hemp is naturally produced, does not require much energy to process, does not require maintenance and consumes $\mathrm{CO}_{2}$ to grow, making the hemp concrete a carbon-negative construction material [1]. Moreover hemp concrete has very interesting properties: it is very light, provides excellent acoustic absorption, thermal insulation and hydric regulation, and prevents condensation. The remarkable physical properties of this material are attributed to its specific multi-scale porous 
structure [2]: a micro-porosity in the hemp shiv and in the mineral matrix (paste), a mesoporosity due to the presence of air in the paste and a macro-porosity due to voids in the binder-hemp packing. However, the exact mechanisms at work behind these properties are not yet well understood. In particular the thermal and mechanical properties of hemp concrete depend on moisture content so that it is critical to follow and understand the moisture transport in hemp concrete [3-4].

Although moisture transfers inside hemp concrete are known to have a significant role on their properties they are poorly understood, in particular because it is hard to have straightforward information on the water distribution inside the sample. The hygroscopic behavior of hemp was the subject of several works [5-8]. In fact it is well known that hemp can rapidly absorb large amounts of water during material preparation (up to 3 times its own weight) but the basic mechanisms of liquid water absorption are not well understood. Then, as cement needs water to hydrate, there can be a competition for water between the binder and hemp shives. This water absorption is at the origin of a lack of workability of the concrete a few minutes after the mix. It might also be at the origin of an incomplete hydration of the binder that is sometimes observed and leads to very poor mechanical properties. However it was also suggested that the incomplete hydration is due to a physico-chemical incompatibility between the binder and the plant aggregate [9]. Another explanation In that case the compression strength of the hemp concrete can drop by a factor ten as compared to the usual value when this effect does not occur. Therefore, we need to understand how water transfers between the different phases of the sample. Such information will also have some interest in other fields where hemp is used although these applications often focus on hemp fibers [1013]. At last it was suggested that liquid water absorption has a greater influence than the vapour diffusion resistance on the moisture and heat transfers in wall components [14].

Previous works in that field are relatively scarce. Usual measurements consist to immerse some hemp in water and take it out and weigh it after different times of immersion [15]. The standard RILEM measurement is based on such an approach, with in addition a centrifugation preceding each weighing. Note that the centrifugation allows removing a part, but not all, of the free water wetting hemp shives (i.e. the water lying along the external surface of the hemp shives), and the remaining amount is unknown, so that there subsists a significant uncertainty on the effective water mass inside the hemp sample which itself forms a porous medium (see below). One study attempted to measure the capillary rise in hemp fibers [16], but it is not 
clear yet whether capillarity effects play the major role in water absorption. However it was also shown that the water absorption in hemp can be influenced by surface treatments [17].

The existing knowledge on water absorption in wood may be helpful for understanding absorption in hemp. The knowledge in that field is more advanced [18] but still complex: there is a large variability in the processes depending on material type, and it appears that wood cannot simply be considered as a homogeneous porous medium in which water would penetrate as a straight front due to capillary effects. Like hemp shives, wood is composed of elongated cells called lumens (i.e. capillaries) which are connected by piths, and water may be found as constitutive, bound or free water. The constitutive water is part of the polymers that constitute the cell walls. Its removal requires high temperatures, which may also lead to the destruction of the material. The bound water is adsorbed inside the cell wall, it is linked by hydrogen bonds to the polymers that constitute the cell wall. Free water corresponds to liquid water inside the lumens. During imbibition of a wood sample, water penetrates in the lumens due to capillary forces and then diffuses in the wall cells under the effect of hydrogen bonds. The penetration of water molecules in the cell walls induces some swelling of the structure.

The question of absorption of water in hemp is complicated by the fact that hemp generally takes the form of a heap of small (centimetric) pieces stacked on each other. As a consequence this is a porous medium with two very different porosities, namely that of the packing and that of each shiv. In the present work, in order to clarify the physical processes occurring during water absorption we study the phenomena at different scales, i.e. that of the packing and that of the shiv. Moreover a simple weighing hardly makes it possible to distinguish the mass of water effectively inside the shives from that just wetting the pieces (i.e. along the external surface of the pieces). ${ }^{1} \mathrm{H}$ NMR relaxometry of water molecules, which is sensitive to the pore size will appear as a useful tool for distinguishing these different phases.

Materials and methods are first described (Section 2), then the result of the monitoring of hemp imbibition obtained by weighing an immersed sample are presented (Section 3.1). The results of NMR relaxometry (Section 3.2) are then compared with data obtained from weighing (Section 3.3), and finally the water penetration through $\mu$ MRI (Magnetic Resonance Imaging) is directly observed (Section 3.4). 


\section{Material and methods}

\subsection{Materials}

117

118 We mainly use a hemp "Chanvribat" provided by BCB (Balthazard \& Cotte Bâtiment)

119 Tradical, which was retted before grinding. Typical particle size is around $1 \mathrm{~cm}$ length and 2

120 mm width. Hemps from various origins were also used to compare our measurement methods

121 with the standard one. They were obtained from three other suppliers: Planet Chanvre,

122 Concrete LCDA, Agrofibre. Hemp shiv is obtained by removing seeds and fibers from the hemp, and the corresponding material is then cut in an industrial device. All four shives used in the present study were directly received from industrial plants and were used as such with no additional treatment. Unless explicitly pointed out, here the hemp is used without preliminary drying. We preferred to follow this approach because drying is known to affect the water absorption properties of wood materials, and the former properties are recovered only after a long time. Here, since our material has been left a long time at ambient humidity (about $50 \%$ on average) and due to the large hygroscopic time scale of such materials we consider that before each test it has reached an equilibrium initial water content of about $10 \%$

131 (this was checked through drying). Such materials will be referred to as dry samples.

132 However we also looked at the impact of a preliminary drying, as will be described below.

\section{3}

134

135

136

137

2.2 Water absorption followed by direct weighing

This test aims at measuring the amount of water entering hemp by observing the change of apparent density of a sample immersed in water. For this measurement, hemp is suspended to a scale and immersed in water (see Figure 1). Hemp shives are compacted in a cage of $8 \mathrm{~cm}$ in diameter equipped with a screw (allowing the compaction). The center of the sample volume is situated around a depth of $6 \mathrm{~cm}$. The resulting sample mass is $40 \mathrm{~g}$ for an apparent volume of 200 to $350 \mathrm{~cm}^{3}$ depending on the imposed pressure. The compaction is such that all hemp shives are in contact with each other, and no shiv behaves loosely, but there still exist small air paths everywhere in the sample so that air escaping from hemp can easily move outside the cage. The cage is made of crossed steel bars leaving holes of about $5 \mathrm{~mm}$. The sample is then suspended via a rigid bar to a scale and immersed in water. And we follow the apparent mass of the sample in time. 
150

$$
V=(K / \mu) \nabla(\sigma \cos \theta / R+\rho g z)
$$

163 In which $\sigma$ is the surface tension of water (here taken as 0.07 Pa.m ), $\theta$ the contact angle and $164 \mu$ the water viscosity $\left(\approx 10^{-3}\right.$ Pa.s $)$. Assuming a perfect wetting $(\theta=0)$ and taking a 165 permeability $K=R^{2} / 12$ (as for parallel cylinders of radius $R=0.5 \mathrm{~mm}$ and of length $4 \mathrm{~cm}$ )

166 and considering that the liquid has penetrated over a distance of $4 \mathrm{~cm}$, i.e. the sample radius, 167

Figure 1. Scheme of the experimental setup used to monitor the water uptake of hemp shives contained in a cage.

The compacted hemp forms a porous system with large pore sizes between the hemp shives. Direct observation of the voids between hemp pieces at the free surface of the sample suggest that the typical pore size inside the medium is of the order of $1 \mathrm{~mm}$, a result consistent with the typical pore size for a packing of compact objects, i.e. a few times smaller than the object size. Let us assume that the dry sample is instantaneously set up at a mean depth of $z=6 \mathrm{~cm}$ in water. To estimate the water penetration velocity $(V)$ in the sample we can apply Darcy's law:

\section{Cage containing} hemp sample

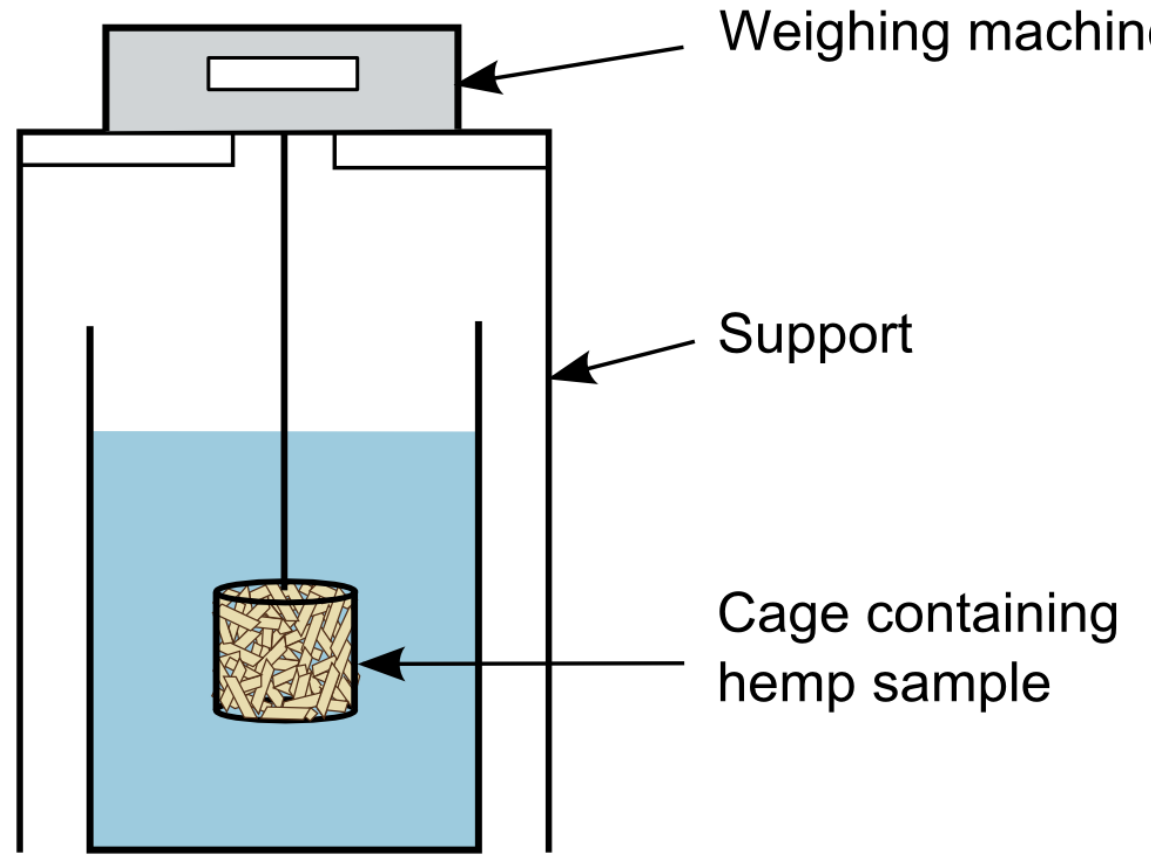


the large pores of the sample (between the shives), i.e. in a time of the order of that needed to fully immerse it. In the following we will assume that this penetration is finished when the other phenomena start to take place.

Now we can look at the typical velocity of penetration in the vessels of the hemp shiv, whose diameters are typically of the order of $50 \mu \mathrm{m}$. Using again (1) with a length of penetration of the order of $1 \mathrm{~cm}$ we find a velocity of the order of $2 \mathrm{~cm} \cdot \mathrm{s}^{-1}$, which means that the penetration through such channels should also be very fast as compared to our time of observation.

Let us now assume that the sample has been immersed and the large pores between the hemps pieces have been filled with water (mass $m_{e x t}$ ). Under these conditions the apparent weight (as measured by the scale) of the cage volume is equal to the effective weight $P_{\text {eff }}=m_{S} g+m_{W} g+m_{\text {ext }} g$ minus the buoyancy force $B=\rho V_{S} g+m_{\text {ext }} g$, in which $m_{S}$ is the mass of solid (i.e. the mass of dry hemp), $m_{W}$ the mass of water which has entered the hemp shives (and replaced air volumes) since the beginning of the test (we neglect the mass of air), $V_{S}$ the total apparent volume of the hemp shives and $\rho$ the water density.

If the hemp shives keep a constant apparent volume, the evolution of the apparent weight will exactly correspond to that of the mass of water entering the hemp shives (and replacing air), i.e. $m_{W}$. If the hemp shives swell as a result of some water entrance we will have a variation of the apparent volume $\Delta V_{S}=m_{a} / \rho_{a}$, in which $m_{a}$ and $\rho_{a}$ are the mass and density of this specific water. Moreover the effective weight will now include an additional term $m_{a} g$, and the apparent mass will finally write: $m_{W}+m_{a}\left(1-\rho / \rho_{a}\right)$.

\subsection{TC RILEM protocol for water absorption}

This test aims at measuring the amount of water absorbed by hemp from the change of apparent mass of a hemp sample. This protocol was performed on various hemps of different origins within the context of RILEM tests. Shives are dried at $60^{\circ} \mathrm{C}$ for this measurement. About $25 \mathrm{~g}$ of hemp are placed in a synthetic permeable bag with holes of approximately 1 $\mathrm{mm}^{2}$ section area and immersed $1 \mathrm{~min}$ in water. The bag is then removed from the water bath and put in a salad spinner which is then rotated 100 times at approximately $2 \mathrm{rpm}$. At the end 
the bag containing shives is weighted and then immersed again for $15 \mathrm{~min}, 4 \mathrm{~h}$ and $48 \mathrm{~h}$,

201 before the same protocol of mangling then weighing is applied. The water absorption capacity of the synthetic bag is independently measured. The measurement is repeated 3 times for each hemp with a standard deviation lower than $15 \%$. Here we only present the average value of

204 the water mass divided by the mass of dry hemp.

\subsection{NMR measurements}

208 Here we intend to follow water absorption from the evolution of NMR signal associated with different pore size in hemp immersed in water. For these measurements, shives had to be grinded in order to enter the experimental set-up. So we first put the hemp in a rotary blade mixer during a short time which allows to get a new shiv size on the order of three times smaller than the initial value. Then this hemp is compacted in a $1 \mathrm{~cm}$ thick layer at the bottom

213 of a $18 \mathrm{~mm}$ diameter tube and water is added until the water free surface reaches the top of the 214 hemp packing. The initial masses of water and dry hemp are measured before introduction in the tube. In our tests the initial water to hemp mass ratio in the tube was always larger than 5, which means that hemp is in contact with more water than it can absorb, even after a long time. In the following description we consider the initial time as that associated to the first contact between hemp and water in the tube.

A Bruker Minispec MQ20 ND-Series, with a $0.5 \mathrm{~T}$ magnetic field corresponding to a ${ }^{1} \mathrm{H}$ (proton) resonance frequency of $20 \mathrm{MHz}$ was used for NMR measurements. The probe (3 $\left.\mathrm{cm}^{3}\right)$ was temperature-controlled $\left(20^{\circ} \mathrm{C}\right)$ by circulating water. The same apparatus was used to perform both $\mathrm{T}_{1}$ and $\mathrm{T}_{2}$ relaxation time measurements.

In order to get the $T_{1}$ distribution, longitudinal proton magnetization decays as a result of the NMR signal relaxation were measured by means of the Inversion Recovery sequence [19], with 50 values of inversion times logarithmically distributed from 0.1 to $12000 \mathrm{~ms}$. Recovery delay was chosen equal to $12 \mathrm{~s}$ to ensure complete relaxation of water between two measurements, which implies a measurement duration of approximately $40 \mathrm{~min}$. The sample is left inside the device and the evolution of $T_{1}$ is followed during a few days after the first contact. 
The $\mathrm{T}_{1}$ distribution can then be resolved by means of ILT (Inverse Laplace Transform). Our

234 procedure is a non-negative least square fit to the data with Tikhonov regularization, is similar to the 'Contin' method [20-21], and is described in [22]. We finally get an apparent statistical distribution of $\mathrm{T}_{1}$, expressed in terms of signal intensity associated with each possible value of $\mathrm{T}_{1}$. Typically such a distribution consists of several peaks situated at different $\mathrm{T}_{1}$ values. Due to the unstable nature and known imperfections of ILT processing [22], such a distribution does not provide a precise description of an effective distribution of $\mathrm{T}_{1}$. Two parameters of this distribution nevertheless constitute relevant physical characteristics of the sample:

(i) The positions of the peak maxima, that we will here call the $\mathrm{T}_{1}$ values. Roughly speaking these values are related to the mobility of water molecules, and specific interactions of water with their environment (e.g. adsorption, proton exchange with other species, or magnetic interactions at nanoscale). In the particular case of water embedded in a pore cavity, within the usual hypothesis of biphasic fast exchange [23], $\mathrm{T}_{1}$ scales as the ratio of the volume of free liquid water to the area of the water-solid interface, with a factor depending on the NMR surface relaxivity. Note that this volume to surface ratio is proportional to radius in the case of uniform spherical pores. Thus the

Measurements of the transverse relaxation $T_{2}$ decays were performed thanks to the CarrPurcell-Meiboom-Gill (CPMG) sequence. The classical sequence was adapted in order to have a varying value of echo time $\tau$. Thus, the value of echo time is incremented at each echo by multiplying the old value of $\tau$ by a low factor. Measurements are done with a recycle delay of $12 \mathrm{~s}$, with 8 scans, with $800 \mathrm{Pi}$-pulses and pulse separation varying from $\tau=2.2 \mathrm{~ms}$ to $5 \mathrm{~ms}$. The acquisition time is about $2.6 \mathrm{~min}$. The relaxation time distributions were extracted using the same ILT processing as for $T_{1}$ distributions [22]. As for $T_{1}$ measurements, each NMR acquisition gives direct information on the quantity of mobile water inside the sample and the $\mathrm{T}_{2}$ distribution. However the resulting data appeared to be rather noisy so that it is difficult to distinguish clear trends. As a consequence for most of the analysis we decided to focus on $\mathrm{T}_{1}$ data. From $\mathrm{T}_{2}$ data, which are obtained in a much shorter time, we could at least deduce that an amount of water of the order of the first value observed from $T_{1}$ measurements (i.e after 
several tenths of minutes of imbibition) is already present inside the hemp after a time as short 268 as $2 \mathrm{~min}$.

\subsection{MRI measurements}

272 Here we intend to have information on water absorption in a hemp shiv from the evolution of

273 the aspect of MR images. The $\mu$-MRI experiments were performed at $17.6 \mathrm{~T}\left({ }^{1} \mathrm{H}\right.$ Larmor 274 frequency of $750.13 \mathrm{MHz}$ ) on a Bruker Avance IIIHD spectrometer equipped with a Bruker 275 Micro 2.5 micro-imaging system $\left(100 \mathrm{G} / \mathrm{cm}\right.$ for the three axis) and a $10 \mathrm{~mm}$ diameter ${ }^{1} \mathrm{H}$ 276 resonator. Acquisition and data processing were achieved with the Bruker Paravision 6.0 277 software. The imaging protocol was carried out using the "Zero Echo Time" sequence (ZTE), 278 [24]) which is particularly dedicated to materials with short transverse relaxation time $\left(\mathrm{T}_{2} *\right)$ 279 such as porous media. 3D images were acquired with a theoretical resolution of $47 \mu \mathrm{m}$ which 280 took from 9 min to $1 \mathrm{~h}$ depending on the number of scans.

282 In order to follow the water absorption in one shiv, we used a NMR tube of $5 \mathrm{~mm}$ diameter. 283 The shiv is chosen so that its width is around $5 \mathrm{~mm}$. Thus it can be jammed in the tube at a 284 given height with a vertical orientation of the fiber. This jamming is sufficient to avoid any 285 displacement of the shiv even in the presence of strong vibrations possibly induced by the 286 application of the pulsed magnetic field gradients during the measurements. Half the tube is 287 filled with water in such a way that the lower half of the shiv is immersed in water (see Figure 288 2). The MRI sequence allows selecting several cross-sectional slices along the main (fiber) 289 axis of the shiv (see Figure 2). Finally we get (see Figure 2 right) the amount of water in a 290 slice of the sample, with mainly two zones: in hemp and in water surrounding the shiv. Since 291 the water level in the tube decreases in time due to water absorption in hemp, the pure water 292 regions that may be seen in the MRI images soon essentially correspond to residual water 293 menisci left around the shiv. At some time the shiv may lose contact with the outside bottom 294 water but we have no clear information about that. 


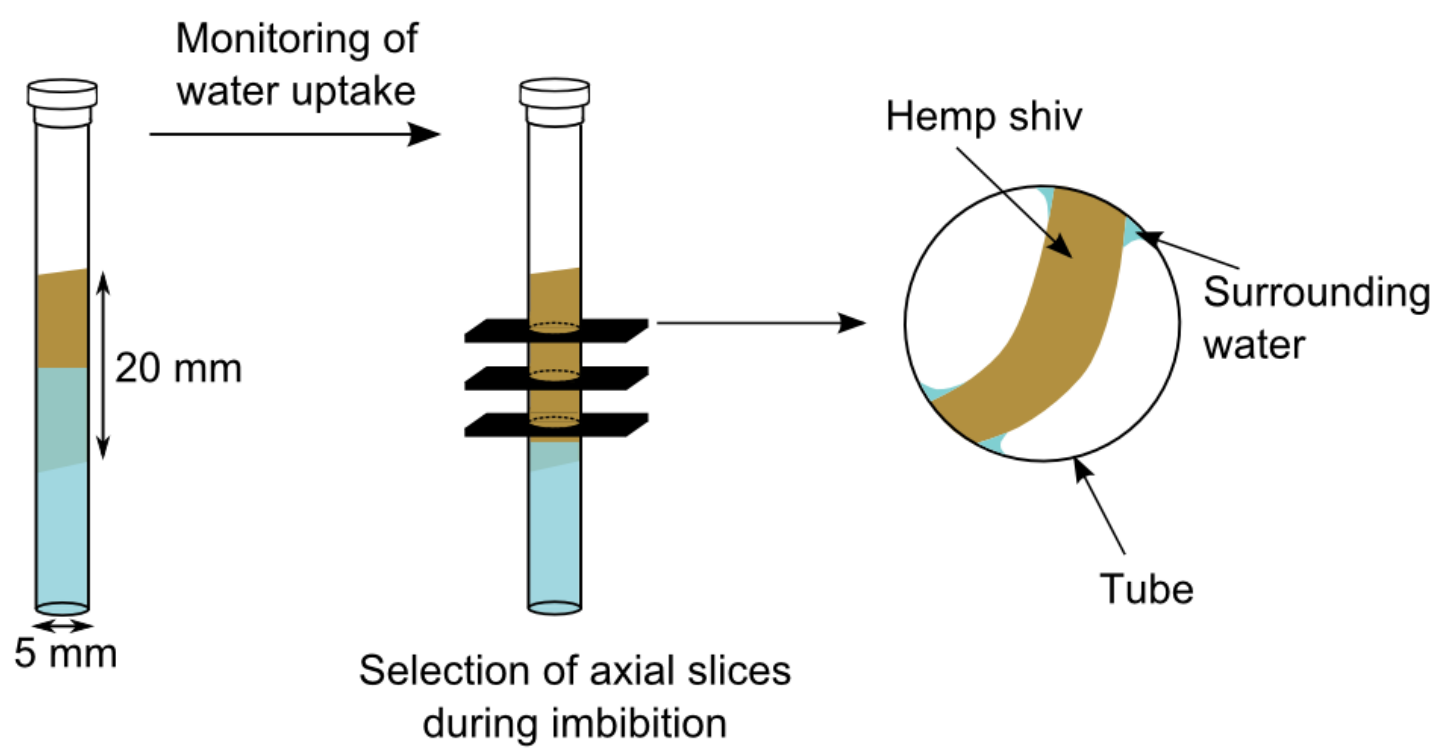

Figure 2. Experimental setup used to perform imaging on one hemp shiv (brown) partially immersed in water (blue) (left) and schematic MRI image (right) resulting from

\section{Results and discussion}

3.1 Weighing of an immersed sample

306 In Figure 3 we represent the evolution in time of the apparent sample mass divided by the initial mass of dry hemp for such an experiment. The typical curve shape in linear scale over several tenths of hours includes a very fast increase during the first minutes followed by a very slow increase in time over several days. The overall shape of the curve is consistent with the observations reported in literature [25]. Repeating several times such a test with different

311 initial hemp volumes we see that there is a significant variability of the result (inset of figure

312 3). This is due to the complex effects which occur over a short time: penetration through the 313 porous hemp shiv network and initial imbibition of the hemp. Ideally, i.e. as assumed in the 314 theoretical treatment above, we would need to have the two processes successively so that we 315 could identify the initial time and initial mass at the beginning of imbibition, but since they 316 occur more or less at the same time this is not possible. Under these conditions in the following we will just comment the shape of the imbibition curve. 
319 Looking at the detailed evolution in a logarithmic scale we see an intermediate period (say between 1 and 10 hours) where the water mass marks a plateau and even tends to slightly decrease during a few hours before starting again to increase significantly. Such a decrease is surprising because it would a priori means that some water now leaves the hemp pores and is replaced by air, but there is no reason to suddenly invert the process of absorption. Another explanation could be that some air bubbles cannot escape easily from the cage and are jammed between (or stuck to) the particles. However such an effect should lead to a plateau of mass, and not a decrease, since it is equivalent to have no more water entrance in the hemp.

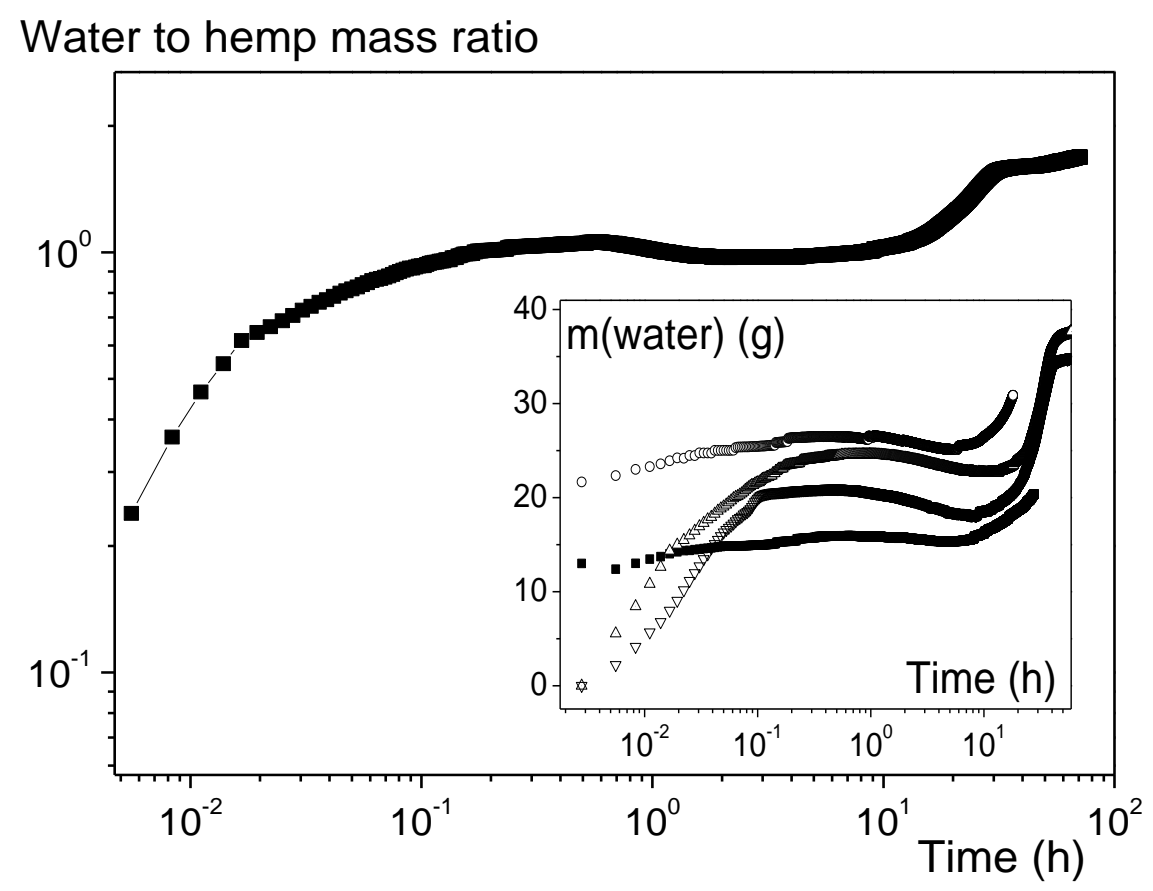

334 Another possibility would be a change of water density after absorption in the hemp. Such an 335 effect was suggested for wood: bound water adsorbs to polymers that constitutes cell walls, and only when cell walls are saturated, liquid water fills the cells. Various investigators tried to measure the void volume fraction of wood by immerging wood in different liquids or gases (helium, benzene and water) and measuring the volume of displaced fluid [26]. Benzene was assumed not to enter microvoids in wood, unlike helium. They observed that in the case of 
wood immerged in helium, the specific volume was lower than for benzene, which confirmed

341 their hypothesis. But in the case of water, the specific volume was even lower than for helium, 342 which means that water does not only enter microvoids. They explain this difference by a 343 compression of water linked to the cell wall by hydrogen bondings. This compression, which 344 corresponds to an increase of water density, might be due both to higher attractive forces in 345 the cell wall than in bulk water and to a reorientation of water molecules. However according 346 to the above equation such a density increase $\left(\rho_{a}>\rho\right)$ would induce an increase of the 347 apparent weight of the sample, and not a decrease as observed.

349 Finally the only explanation seems to be that there is a kind of transformation of some liquid 350 in gas. Actually such an effect can be observed as soon as water is put in a solid container. 351 After some time one can observe the formation of small bubbles attached to solid surfaces 352 (see for example tap water in a glass). This results from a degassing of solubilized gases (e.g. $\left.353 \mathrm{O}_{2}, \mathrm{CO}_{2}\right)$ at the contact with a solid surface. If the small bubbles thus created remain attached 354 to the shives the hemp sample will appear lighter after some time, as observed in our tests (see 355 Figure 3). Unfortunately we did not find any solution to avoid or control this effect: some air 356 in contact with the liquid may dissolve in it during the test; and removing the ambient gas will 357 tend to accelerate the imbibition.

\subsection{Monitoring using NMR}

The weighing of an immersed hemp sample does not allow to get fully reliable data concerning the mass of water entering the pores. Therefore, we use NMR to measure the kinetics of water absorption in hemp.

\subsubsection{Various types of water}

368 As we have seen in the introduction three types of water may be considered in wood. Since 369 depending on their environment and the interactions they develop, the spins of the water 370 hydrogen protons exhibit different NMR relaxation times, these different types of water may 371 exhibit different relaxation times. For example, according to Almeida [27], for Douglas fir the 372 transverse relaxation time $\left(\mathrm{T}_{2}\right)$ of constitutive water is of the order of a few tens of 
373 microseconds, that of bound water ranges from one to a few milliseconds, and that of water in 374 the lumen ranges from ten to a few hundred microseconds.
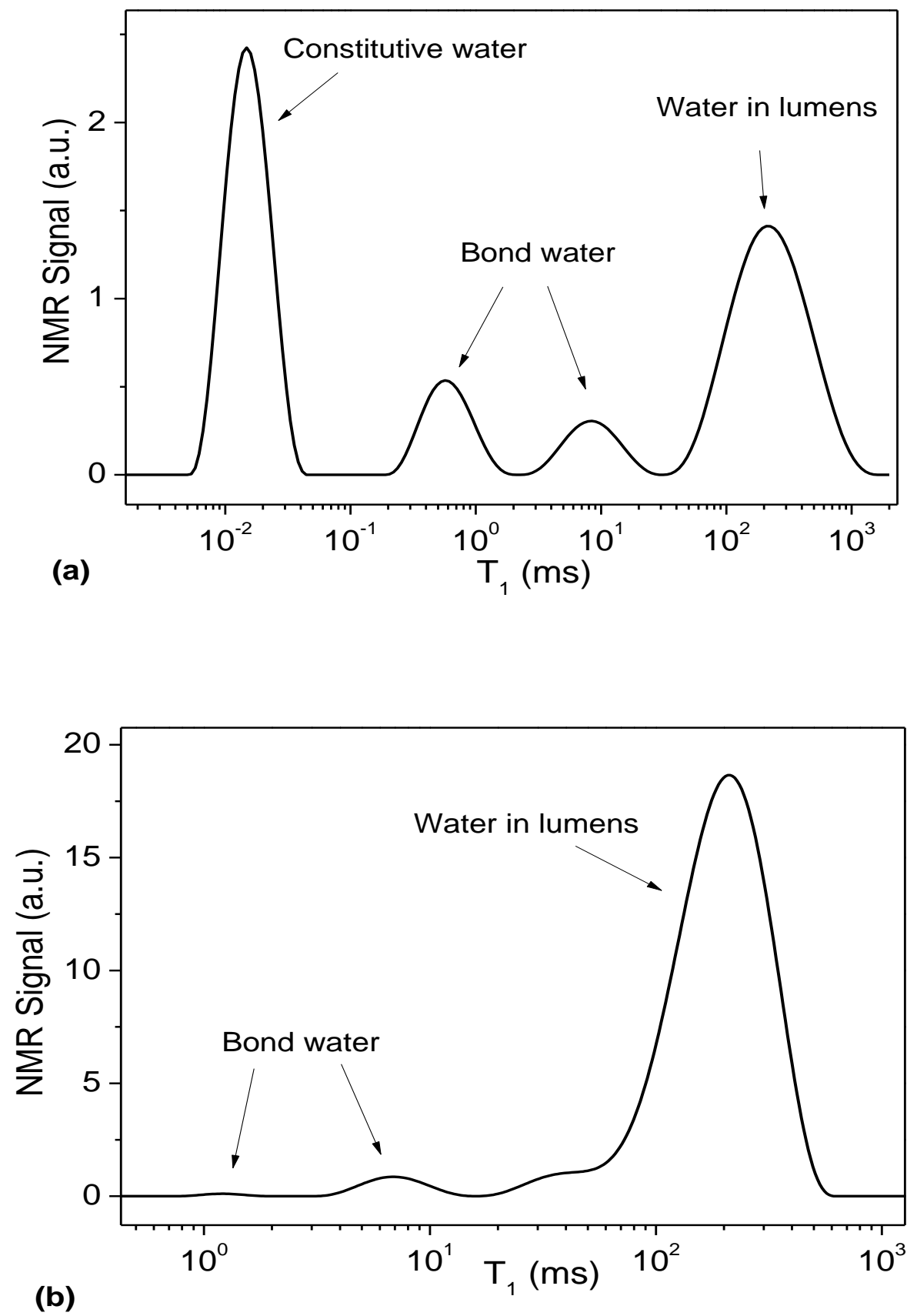

Figure 4: Distribution of relaxation times for hemp shives under ambient conditions (a) and after immersion in water for $2 \mathrm{~min}(\mathrm{~b})$

383 The distributions of $\mathrm{T}_{1}$ relaxation time for dry hemp and moisten hemp ( 2 min immersed 384 sample in water) are presented in Figure 4. Extrapolating the above analysis of water state in 
wood to this case we can suggest that the peak associated with the smallest relaxation time corresponds to constitutive water, the two central small peaks to bound water and the right peak (i.e. the largest relaxation time) to water in lumens. It is interesting to note that even in this state (hemp under ambient conditions) the signal associated with the three states of water is significant, whereas we could have expected to have negligible water in lumens. However we cannot compare the signal intensity in these three states to that found during absorption because the absolute signal level for two different tests (and different samples), even with the same water amount, generally varies.

When the hemp shives have been immersed in water for a few minutes (see Figure 4b) the distribution of relaxation strongly differs from that for the "dry" sample. Here we focused on longer relaxation times so that we did not record the signal associated with the constitutive water. The main peak is now the one associated with liquid water in the lumens. We can also remark that the peak associated with the largest relaxation time for bound water has significantly increased with regards to the other peak (lowest relaxation time) for bound water. This illustrates the fact that most water has entered the lumens and a small part of it entered the cell walls but only in the largest pores of these cells.

\subsubsection{Monitoring water absorption $\left(\mathrm{T}_{1}\right)$}

We now consider the situation where we have the hemp sample immersed in an excess of (outside) water for a longer time (see procedure in Section 2). The distribution of relaxation times of this system now exhibits two main peaks (see Figure 5), associated with two types of environment. The shortest relaxation time, around $300 \mathrm{~ms}$, is close to the relaxation time of protons in liquid water that we observed previously in moisten hemp. Therefore, it is associated to water in the lumens. The peak corresponding to relaxation times around $2 \mathrm{~s}$ corresponds to water outside the hemp. At last we still have a small peak at $\mathrm{T}_{1}$ below $100 \mathrm{~ms}$ which is associated to water bound in hemp, but the signal intensity associated with this water state is much smaller than that in the lumen.

As the area under each peak is proportional to the amount of water in each type of pore, we can monitor the amount of water that entered in hemp over time. In Figure 5, we can already note that the area beneath the curve part corresponding to water outside hemp seems to decrease while the area associated to water in hemp seems to increase, which corresponds to 
419 the transfer of water from outside to inside the hemp shives. Note that the values of the 420 relaxation times associated with each peak slightly change during the process but such 421 changes may be attributed to a side effect of ILT processing, which can shift neighbouring 422 peaks depending on their relative amplitudes [22].

423

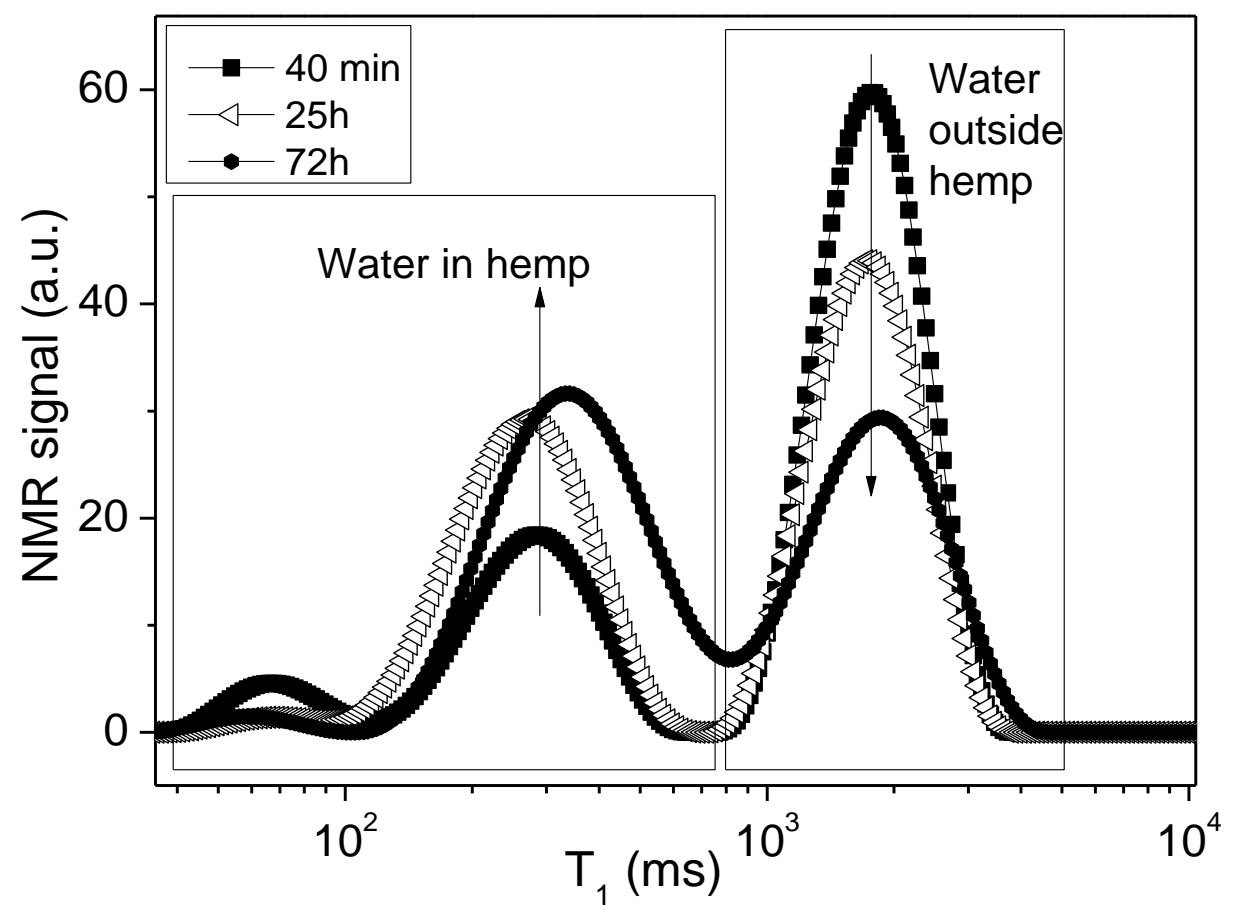

Figure 5: Distribution of $T_{1}$ relaxation times for a hemp sample put into contact with an excess of 426 water, at three times during the test. 


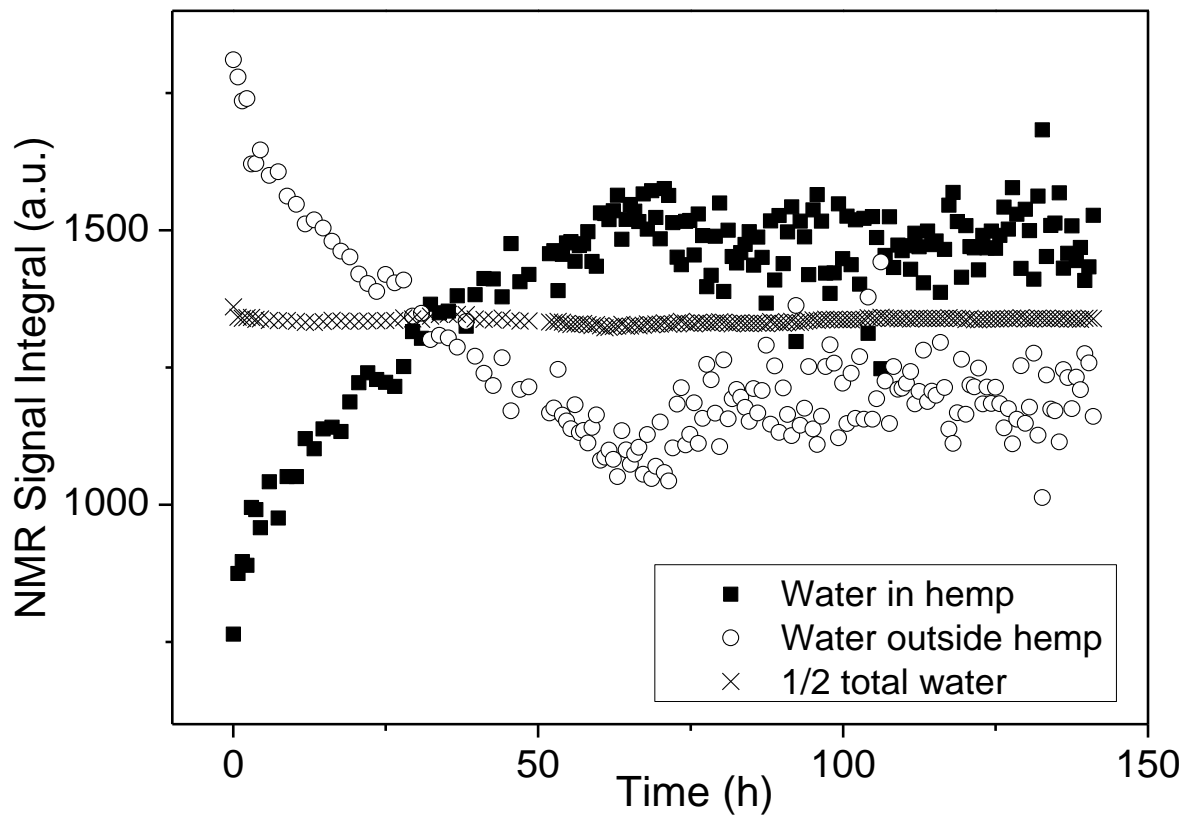

Figure 6: Evolution of the signal intensities associated to water in hemp and water between hemp particles over time. Half of the total water amount in the sample is also represented.

432 In Figure 6 we show the evolution of the integrated signal for water inside hemp and outside 433 hemp. We see that the signal associated to water in hemp increases in time while the signal 434 associated to water outside hemp decreases. The consistency of this type of measure is 435 demonstrated through the fact that the total water amount deduced from these data remains 436 constant (see Figure 6), as expected since the tube is closed. This in particular confirms that 437 we effectively record the water entering hemp with this technique. We can also notice that the 438 initial signal associated to water in hemp is ten times higher than the signal measured for dry 439 hemp. This confirms that hemp absorbs a significant amount of water during the first 440 measurement. 


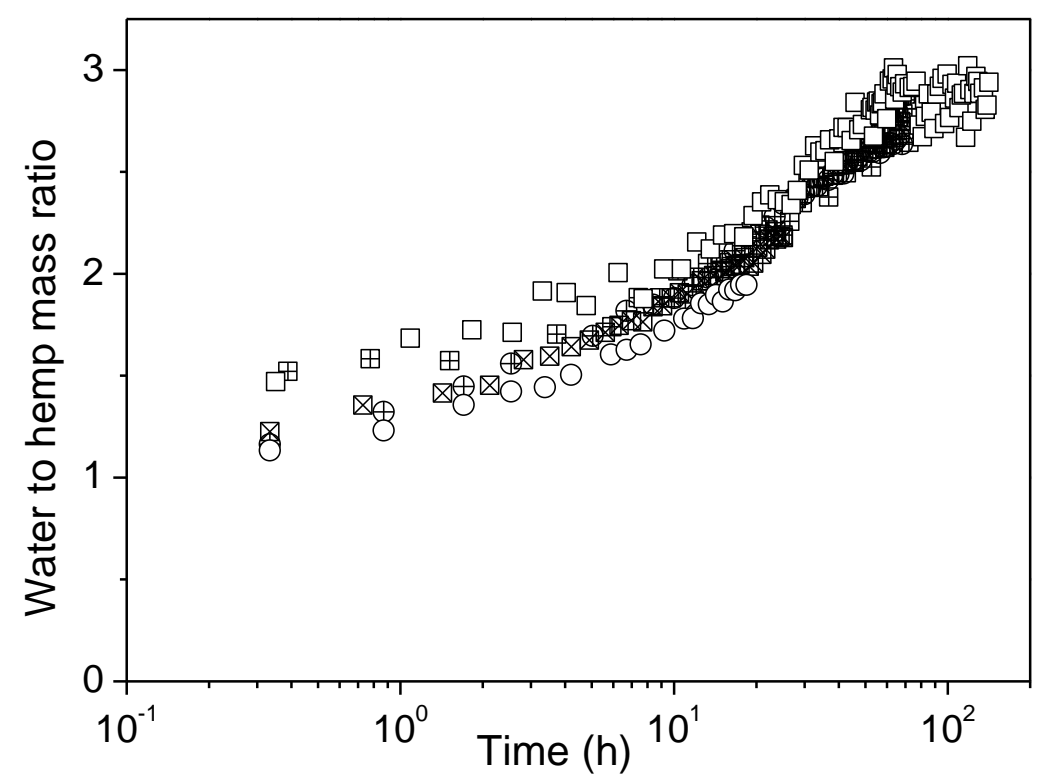

441

442

443

444

445

446

447

448

449

450

451

452

453

454

455

456

457 We also tested the impact of a preliminary drying of the sample which, as already mentioned, 458 459

Figure 7: Water to hemp mass ratio measured by NMR for different samples (either crossed or not) either dried (circles) or not (squares). For the dried samples the water to hemp mass ratio was computed by adding to the hemp mass, and substracting from the total water mass, the mass of water lost by drying, so that the data presented here for dried and non-dried samples can be relevantly compared.

The mass of water that is added to hemp is weighted as the sample is prepared. Knowing this mass allows to calculate the effective amount of water in each phase (inside or outside hemp), and particularly to determine, for each test, the (linear) relationship between the NMR signal intensity and the water to hemp mass ratio over time. From such measurements we now have a view which is in principle not affected by any artefact (in contrast with weighing). The uncertainty on such data is about $12 \%$, as appears from the repetition of similar tests (see Figure 7).

typically withdraws a mass of water equal to about $10 \%$ the hemp mass. It appears that this does not change the general trends of the imbibition (see Figure 7): the data obtained after drying can be transformed in data equivalent to those without drying by adding to hemp the additional mass of water associated with drying, and withdrawing this additional mass of water from the apparent water; the two sets of data appear to be similar to each other within 
463

464

465

466

467

468

469

470

471

472

473

474

475

476

477

478

479

480

481

482

483

484

485

486

487

488

489

490

491

\section{6}

the uncertainty on such experiments. Thus it seems that drying has no more effect than simply shifting the initial water mass towards a lower value but this water loss is balanced by a very fast water imbibition at the beginning of the test.

We see that the hemp has initially (i.e. during the time needed for the first NMR measurement) absorbed a large amount of water of about $150 \%$ of the hemp mass. Then it goes on absorbing water during 3 days, until reaching a mass to hemp ratio of about 3 (see Figures 7 and 8). This confirms that hemp imbibition occurs in two phases each of them associated with the absorption of half the total absorbed water: one over short time, i.e. in less than two minutes (as appears from $\mathrm{T}_{2}$ measurements and weighing), and the other over several days. The first phase likely might correspond to some kind of capillary imbibition in the largest pores, but the entered water does not increase as predicted by a simple Washburn imbibition (i.e. as $\sqrt{t}$ ) [28]. In the second phase the mass increase is much slower (i.e approximately proportional to $t^{1 / 8}$ ). However if we consider that in phase 2 the water simply penetrates in a dry region we could expect a Washburn process for the additional water mass (from the penetrated amount at the end of phase 1, i.e. 1.5). And indeed the additional water mass evolves as $\sqrt{t}$ (see inset of figure 8). Actually, anticipating on results of Section 5 this interpretation is in disagreement with MRI observations: during phase 1 the water effectively rapidly invades a first region of each hemp shiv, but during phase 2 it seems to penetrate globally in another region of shiv, instead of progressively invading it from one side. This suggests that in phase 2 there is a slow diffusion process through the hemp structure rather than a capillary penetration.

We also remark (see Figure 8) that the data from NMR do not show a slight water mass decrease between 1 and $10 \mathrm{~h}$. Instead we now have a slow but regular increase, which suggests that the direct mass measurement of a hemp sample fully immersed in water can be affected by some artefact inducing a significant error on measurements. In the following we will use the NMR data as the reference data for describing the effective water amount absorbed in hemp. 


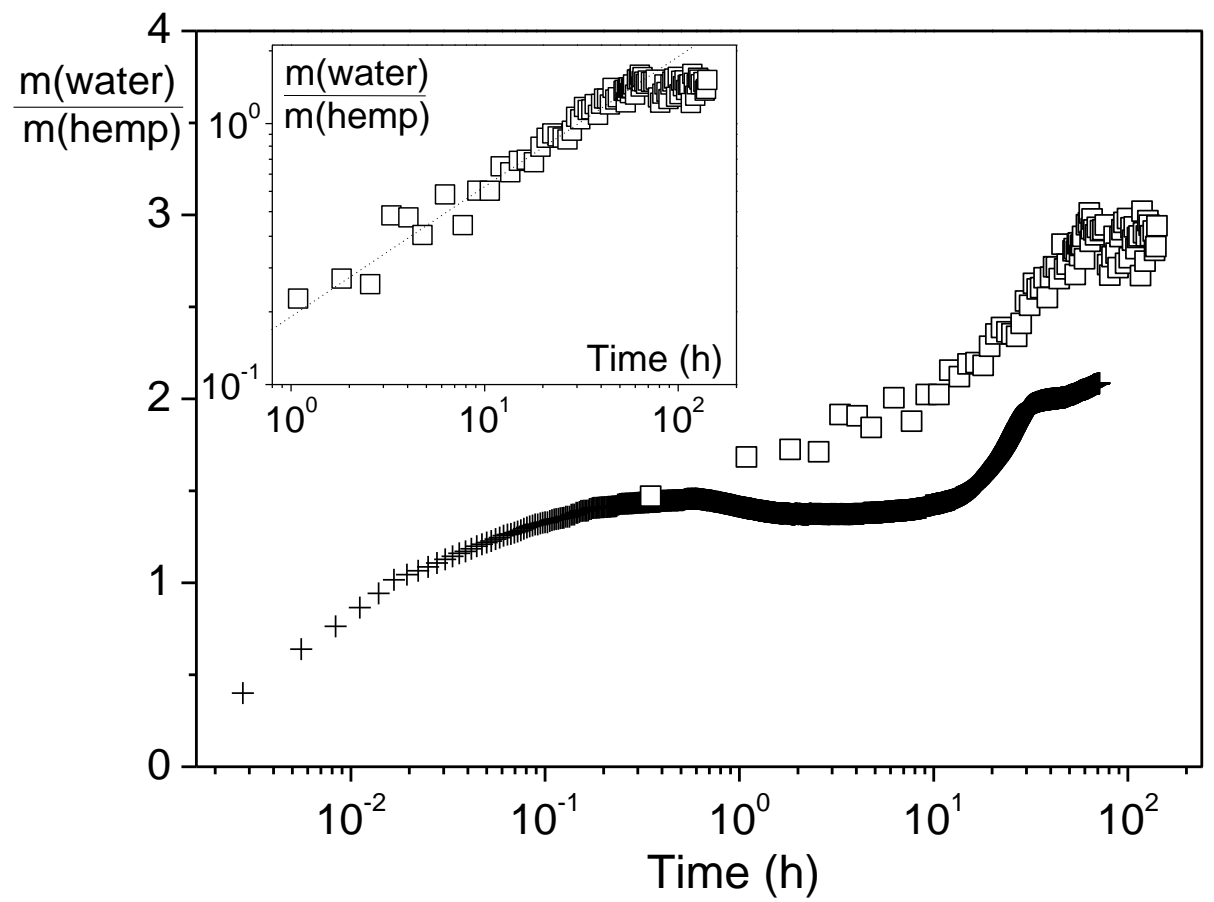

492

Figure 8. Water to hemp mass ratio in time as measured by $\mathrm{T}_{1}$ relaxometry (squares) and by weighing of an immersed sample (crosses). The inset shows the additional (see text) water to hemp mass ratio in phase 2 of imbibition as a function of time in logarithmic scale. The continuous line has a slope $1 / 2$.

\subsubsection{Impact of sample history}

Successive imbibition tests were carried out by drying the sample (put in an oven at $60^{\circ} \mathrm{C}$ until mass stabilization) after each test. By monitoring by NMR the successive water uptakes we see that the second imbibition is different from the first one (see Figure 9). The water mass in hemp increases faster after the first cycle (imbibition + drying), but finally reaches the same saturation level. In the next cycle the water absorption process is the same as the second one. This suggests that the structure has been irreversibly modified during the first cycle but is no more modified in the next cycles. This might be related to the observation that after the first drying (and not in the subsequent cycles) a brown layer overlays the sample, which likely corresponds to the extraction of molecules from the hemp possibly playing a role in the initial imbibition. We also could see that after the first cycle the sample volume has slightly increased so that it tends to be strongly jammed in the container in which it was initially just 
513 packed by hand, which confirms some slight irreversible changes in the structure after the first 514 imbibition.

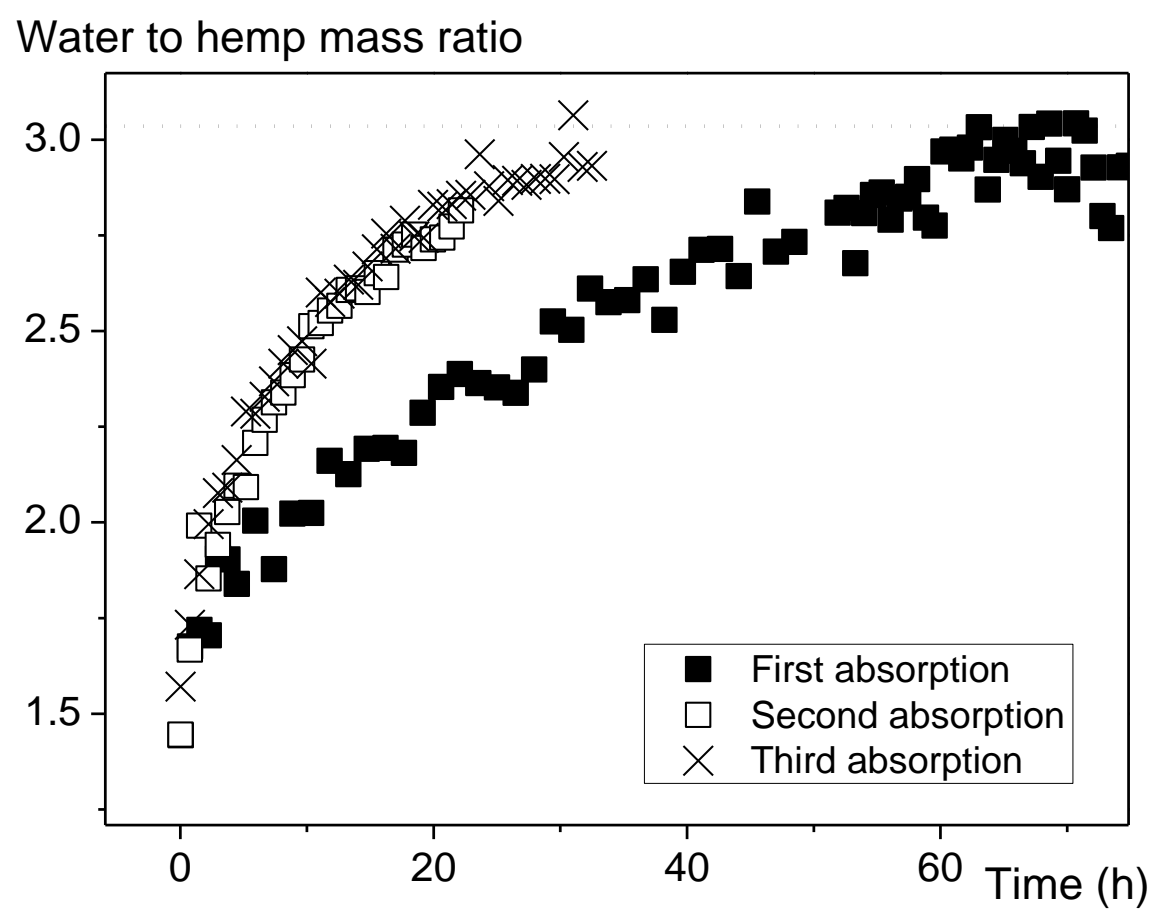

Figure 9: Results of the water absorption in hemp shives during successive imbibitions (separated by drying) measured by $\mathrm{T}_{1}$-relaxometry.

524 We can now compare the results obtained from the TC RILEM method to those obtained by 525 NMR for the four different hemps at different times during the imbibition process. Except for one material the mass found by the TC RILEM method is significantly higher than that found by NMR all along the process (see Figure 10). In fact we have a constant shift of the two evolutions, i.e. at any time during the process the water mass found by the TC RILEM method is just simply equal to that effectively entered in the hemp plus a constant term. It is likely that this term exactly corresponds to the water amount remaining at the surface of the hemp shives

531 due to capillary effects. This would give an additional mass of water proportional to the 532 specific (external) surface of the hemp sample, thus proportional (for a given hemp type) to the mass of hemp by a factor $\alpha$, and consequently an additional term $\alpha$ in the representation 
of $m_{W} / m_{S}$, in agreement with our data. This factor finally appears (see Figure 13) to vary in a 535 rather narrow range (0.4-0.6) depending on hemp type.

537

546 In order to understand why hemp imbibition takes place in two phases with very different 547 kinetics, a hemp shiv imbibition is directly observed using $\mu$-MRI. We used the ZTE

Figure 10: Water to hemp mass ratio measured by the TC RILEM method as a function of that measured by NMR measurements for different hemps at different times during imbibition. The lines have a slope 1 , the continuous line would correspond to a perfect agreement between the two types of measurement.

\subsection{Imaging of water absorption} sequence [24] which allows detecting protons that have relaxation times as short as a few tens of microseconds. Therefore, we expect to detect both bound and liquid water. With this sequence, the signal intensity is proton density weighted, which means that it is proportional to the local amount of protons. As a consequence, roughly speaking the bright parts of the images corresponds to the zones with the highest water density.

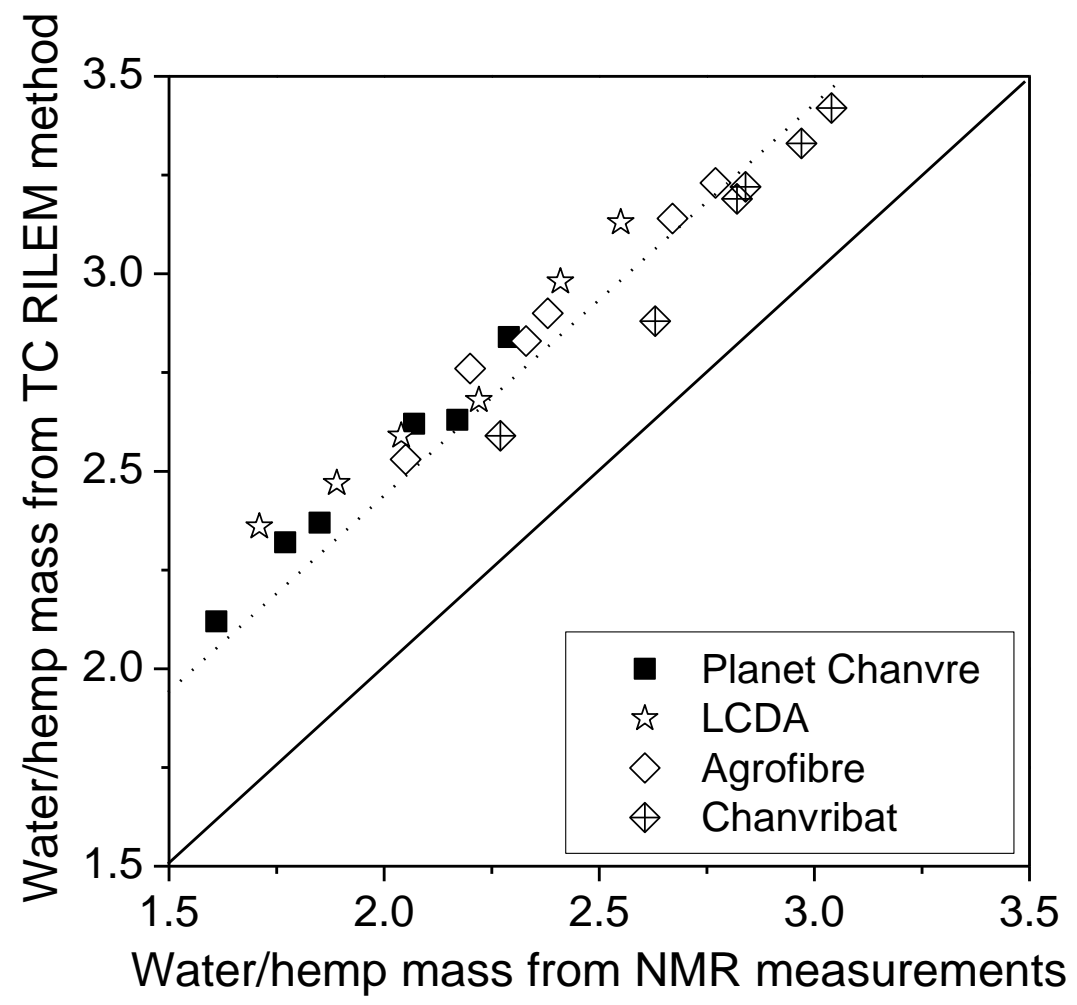


554 We first attempted to get an image of dry hemp but we were unsuccessful, the signal was too

555 low. Images could be obtained when the shiv is in contact with water. In that case the image evolution is qualitatively similar in the different slices along the shiv axis (see Figure 2). Here we focus on one of the central slice to discuss the typical trends. The first image corresponds to signal acquisition during the first four minutes of hemp imbibition. We can see that it is blurred (see Figure 11), which is likely a result of the significant evolution of the water density distribution over the time of acquisition. Indeed in MRI each information at a local scale is not simply the result of the average signal over the total time of acquisition as for several other imaging techniques, here it is based on a reconstruction by Fourier transform of the information obtained from a virtual picture obtained in the field gradient (reciprocal) space. As a consequence significant evolution of the spatial density can lead to this kind of blurring. We can nevertheless still distinguish the contour of the shiv and external water between the shiv and the tube, which are regions in which the water amount changes likely remain negligible.

In the next step, i.e. the image obtained after $18 \mathrm{~min}$, the picture is better resolved, likely because as we have seen in previous measurements the absorption kinetics during that period is much slower than in the first stage. We can now clearly see the hemp shiv surrounded by water. Moreover the shiv seems to be composed of two parts. The left part is much brighter than the right part. These regions correspond to two different tissues, respectively the pith and the wood. The pith is the youngest part of the stem, it is in its centre. During the plant growth, the pith becomes wood through a lignification process. Lignin forms a three dimensional network that provides its rigidity to the cell wall. It also makes the tissues more hydrophilic. In fact, polysaccharide components of the plant cell wall are highly hydrophilic and thus permeable to water. Lignin makes it possible for the tissue to conduct water efficiently [28]. As a consequence, pith is very hydrophilic whereas the woody part is more hydrophobic. This explains that after $18 \mathrm{~min}$, the pith is already very bright whereas the wood does not seem to contain much water. We can also observe some bright points in the woody part of the shiv. They are likely due to some vessels filled with water (which finally represents a small water amount). Indeed the imbibition of wood is known to take place in two successive steps. During a first stage water penetrates through some vessels due to capillary forces. Then it diffuses through vessel walls as a result of hydrogen bonding. Only when these walls are saturated water can stay in the lumens. 
During the next hours, we observe a progressive brightening of the woody part. After $16 \mathrm{~h}$ it seems to contain almost as much water as the pith. During that period the water amount in the pith does not seem to evolve significantly. This suggests that the two successive steps in the kinetics of water absorption in hemp as observed from NMR tests results from the successive imbibition of the two different parts of the shiv: first a fast penetration of water in the pith,

593 then a much slower penetration in the wood.

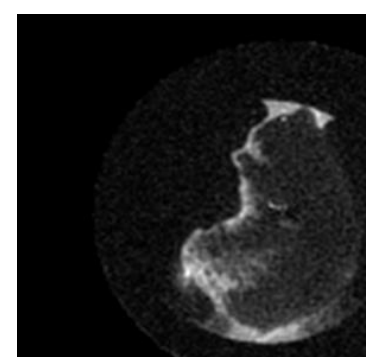

First measurement

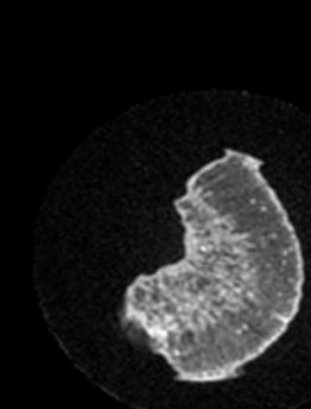

3h11

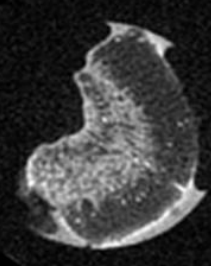

$18 \mathrm{~min}$

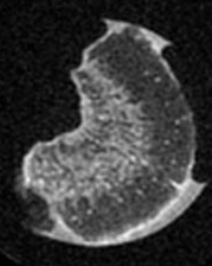

$37 \mathrm{~min}$

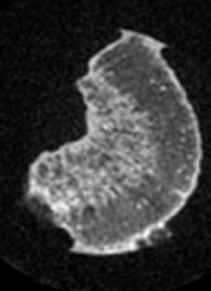

$2 \mathrm{~h} 17$

Figure 11: Evolution of the middle slice of hemp during water absorption as seen by MRI.

\section{Conclusion}

601

602 We have shown that following the evolution of the distribution of NMR relaxation time 603 makes it possible to quantify the effective amount of water entering the hemp. It is more 604 difficult to use the weighing technique to get relevant data and we were unable to understand 605 the exact origin of some artefacts observed in that case, in particular those leading to a water 606 mass decrease while NMR data show a continuous increase of water mass inside the hemp.

608 We also showed that in general the TC RILEM method significantly overestimates the 609 effective water content inside the hemp, an effect likely due to some water remaining along 
610 the hemp shiv surfaces as a result of capillary effects. It was also shown that this

611 overestimation is poorly dependent on the hemp type and does not vary in time (i.e. during

612 absorption), so that it can simply be taken into account for a better interpretation of the results

613 of such tests.

614

615 As clearly seen from NMR, water intake occurs in two steps, a fast one taking place in less 616 than two minutes, corresponding to water entering the pith, and a slow one, taking place over 617 days, corresponding to water diffusing into the woody part of the shiv. Further studies could 618 focus on how plant-related parameters (species, growth conditions...) affect the water intake.

619 The effect of additional treatment, like retting could also now be fully assessed. Finally, the 620 way this two-steps kinetics affect the setting of hemp-lime concrete can now be thoroughly 621 studied and taken into consideration in the formulation of hemp concrete relationship / 622 performance.

\section{Acknowledgement}

Financial support from the TGIR-RMN-THC Fr3050 CNRS for conducting part of this research is gratefully acknowledged.

\section{References}

[1] Boutin MP, Flamin C, Quinton S, Gosse G. Analysis of life cycle of 1. thermoplastic compounds loaded with hemp fibers, and 2. hemp concrete wall on wood structure. French Ministry of Agriculture-INRA Report, MAP 04 B1 0501 (2005) (in French)

[2] Collet F, Bart M, Serres L, Miriel J, Porous structure and water vapour sorption of hempbased materials, Construction and Building Materials, 2008;22:1271-1280

[3] Samri D, Arnaud L, Assessment of heat and mass transfers in building porous materials, Proc. of the $4^{\text {th }}$ European Conference on Energy Performance \& Indoor Climate in Buildings, Nov 2006, Lyon.

[4] Tolkovsky A. Sorption behaviour of hemp and lime concrete floors, MSc Architecture: Advanced Environmental and Energy Studies, Graduate School of the Environment, Machynlleth, UK (2010)

[5] Collet F, Chamoin J, Pretot S, Lanos C, Comparison of the hygric behaviour of three hemp concretes, Energy and Buildings, 2013;62: 294-303 
[6] Bart M, Moissette S, Oumeziane YA, Lanos C, Transient hygrothermal modelling of coated hemp-concrete walls, European Journal of Environment and Civil Engineering, 2014;18:927-944

[7] Walker R, Pavia S, Moisture transfer and thermal properties of hemp-lime concretes, Construction and Building Materials, 2014;64:270-276

[8] Othmen I, Poullain P, Leklou N, Moisture transfer in three hemp concretes, Construction Materials and Structures (ed. By Ekolu SO, Dundi M, Gao X, 1st International Conference on Construction Materials and Structures, Johannesburg), 2014:1358-1363

[9] Diquelou Y, Gourlay E, Arnaud L, Kurek B, Impact of hemp shiv on cement setting and hardening: Influence of the extracted components from the aggregates and study of the interfaces with the inorganic matrix, Cement and Concrete Composites, 2015;55:112-121

[10] Mustata A, Mustata FStC, Moisture Absorption and Desorption in Flax and Hemp Fibres and Yarns, Fibres and Textiles in Eastern Europe 2013; 21: 26-30.

[11] Shahzad A, Effects of Water Absorption on Mechanical Properties of Hemp Fiber Composites, Polymer Composites, 2012;33:120-128

[12] Rouison D, Couturier M, Sain M, MacMillan B, Balcom BJ, Water Absorption of Hemp Fiber/Unsaturated Polyester Composites, Polymer Composites, 2005;26:509-525

[13] Dhakal HN, Zhang ZY, Richardson MOW, Effect of water absorption on the mechanical properties of hemp fibre reinforced unsaturated polyester composites, Composites Science and Technology, 2007;67:1674-1683

[14] Evrard A, Flory-Celini C, Claeys-Bruno M, De Herde A, Influence of liquid absorption coefficient on hygrothermal behaviour of an existing brick wall with Lime-Hemp plaster, Building and Environment, 2014;79:90-100

[15] Stevulova N, Cigasova J, Purcz P, Schwarzova I, Long-Term Water Absorption Behaviour of Hemp Hurds Composites, Chemical Engineering Transactions, 2014;39:559564

[16] Pejic BM, Kostic MM, Skundric PD, Praskalo JZ, The effects of hemicelluloses and lignin removal on water uptake behavior of hemp fibers, Bioresource Technology, 2008;99:7152-7159

[17] Stevulova N, Cigasova J, Purez P, Schwarzova I, Kacik F, Geffert A, Water absorption behavior of hemp hurds composites, Materials, 2015;8:2243-2257

[18] Siau JF, Transport processes in wood, Springer-Verlag, Berlin, 2004

[19] Callaghan PT, Principles of nuclear magnetic resonance microscopy, Clarendon Press, Oxford, 1991.

[20] Whittall KP, MacKay AL, Quantitative interpretation of NMR relaxation data, J. Magn. Reson. 1989;84:134-152. 
702 [21] Provencher SW, A constrained regularization method for inverting data represented by linear algebraic or integral equations, Comput. Phys. Commun. 1982;27:213-227.

[22] Faure P, Rodts S, Proton NMR relaxation as a probe for setting cement pastes, Magn. Reson. Imaging 2008;26:1183-1196.

[23] Philippot S, Korb JP, Petit D, Zanni H, Analysis of microporosity and setting of reactive powder concrete by proton nuclear relaxation, Magn. Reson. Imaging 1998;16:515-519.

[24] Weiger M, Pruessmann KP, MRI with Zero Echo Time, Encyclopedia of Magnetic Resonance, 2012, John Wiley and Sons

[25] Arnaud L, Gourlay E, Experimental study of parameters influencing mechanical properties of hemp concretes, Construction and Building Materials, 2012;28:50-56.

[26] Stamm AJ, Hansen LA, The bonding force of cellulosic materials for water (from specific volume and thermal data). Journal of Physical Chemistry, 1937;41:1007-1016.

[27] Almeida G, Leclerc S, Perre P, NMR imaging of fluid pathways during drainage of softwood in a pressure membrane chamber. International Journal of Multiphase Flow, $2008 ; 34: 312-321$.

[28] Washburn EW, The dynamics of capillary flow. Physical Review, 1921;17:273-283

[29] Sarkanen KW, Ludwig CH, Lignins: occurrence, formation, structure and reactions. Wiley, New York, 1971. 